\title{
The force behind the prize
}

\section{The Quantum Quark \\ by Andrew Watson \\ Cambridge University Press: 2004. 476 pp. \\ $\$ 30, £ 19.99$}

\section{John Ellis}

The Nobel Prize for Physics in 2004 was awarded to David Gross, David Politzer and Frank Wilczek for their discovery in 1973 of 'asymptotic freedom', the key ingredient in the underlying theory of the strong nuclear force. Journalists have had a hard time trying to explain this abstruse breakthrough to the public. Andrew Watson's book The Quantum Quark is probably not cut out for coffee-table stardom, but it is a very complete account of the ideas and experiments that led to the development of this theory and its verification. As such, it may appeal to anybody willing to take the time and make the effort to understand the basis for last year's prize.

Many of the 'usual suspects' of twentiethcentury physics parade through the pages of this book: quantum mechanics, relativity, quantum field theory, and so on. But they are marching towards a specific goal, namely their roles in quantum chromodynamics (QCD), the underlying theory of the strong interactions. It is not every day, nor even every century, that a new layer of the 'cosmic onion' is revealed, so the discovery of the underlying quark structure of nuclear particles and the quantitative understanding of the strong interactions must rank among the highest intellectual achievements of twentieth-century physics.

However, QCD is not a glamorous theory and has not made as big a splash as the unified theory of the weak interactions, with its flashy discoveries of the $\mathrm{W}$ and $\mathrm{Z}$ particles and the long anticipation of the enigmatic Higgs boson. Nevertheless, QCD has great inner beauty, is just as much a part of the standard model of elementary particles, and was from some points of view a tougher theoretical nut to crack.

Watson certainly gives us plenty of theoretical red meat to sink our teeth into, perhaps more than some delicate stomachs can accommodate. As well as being very complete, this book is also reasonably accurate. However, I did notice one scientific howler: electromagnetism is not described simply by the $U(1)$ part of the electroweak theory, but is a more complicated mixture of its different components. Also, I am concerned that the lay reader might find its early sections challenging: the explanations are reasoned and self-contained, but veer towards the succinct.

Personally, I would also have preferred more experimental sauce in this early part.
After all, experiment is the final arbiter of whether nature has the good taste to follow our theoretical ideas. It might have been good, for example, to explain what it was about the photoelectric effect that led Einstein to postulate the existence of the photon, the prototype force-carrying boson that was joined later by the gluons that bind the quarks, and by the $\mathrm{W}$ and $\mathrm{Z}$ particles responsible for the weak interactions. Nevertheless, this introduction and review of basic ideas in field theory and particle physics makes a good appetizer.

Unfortunately, I found the main course unsatisfactory in several respects. First, the scientific events are often not presented in
These topics are all discussed, but not with as much attention and emphasis as I should have liked. Conversely, some more controversial aspects of QCD, such as the everlasting hunts for glueballs and exotic bound states, receive more attention than they may yet deserve. Another gripe is that the book is probably too compendious for many readers, who may get lost in a forest of miscellaneous tree diagrams. I think it would have benefited from more active editing.

So do I recommend this book? The author and his publishers are certainly to be congratulated on their uncanny timing. It is a real coup to have released this book just at the moment when physics students, scientists in

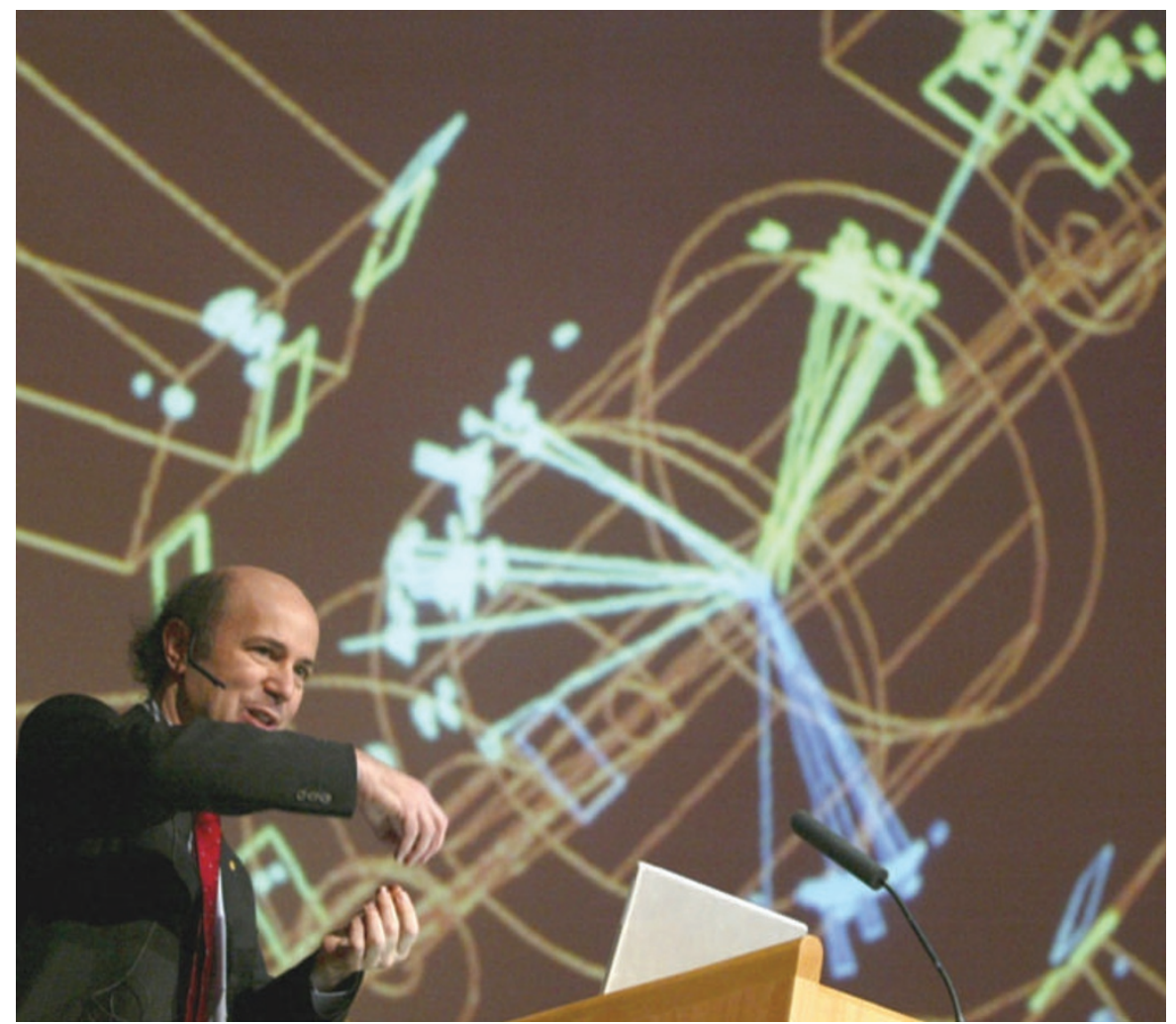

Attractive idea: Frank Wilczek won a Nobel prize for work on the theory of the strong interaction.

chronological order. This might sometimes be a useful literary device, but why discuss deep-inelastic scattering experiments before the earlier pioneering ones that revealed quark partons, or the even earlier ones demonstrating that the proton was not a simple point-like object? There are also some irritating factual errors in this more experimental part. For example, the lower limit on the lifetime of a proton is $10^{29}$ years rather than $10^{25}$ years, the lower limit on the mass of the Higgs boson is $114 \mathrm{GeV}$ rather than 65 $\mathrm{GeV}$, and the maximum energy achieved by the Large Electron-Positron accelerator at CERN was $208 \mathrm{GeV}$ rather than $192 \mathrm{GeV}$.

A more basic shortcoming of the book, in my opinion, is its failure to bring out some of the beauties of QCD, such as the renormalization group and asymptotic freedom, or confinement and the nature of the vacuum. other fields, and general readers around the planet may be keen to unravel for themselves the context for this year's physics Nobel. Given this motivation, they may find this book an informative and fascinating read.

Moreover, although it has some flaws, this book does not have much competition. Andrew Pickering's Constructing Quarks (Edinburgh University Press, 1984) is a real page-turner and is scientifically accurate, but is marred by its aberrant initial and final chapters full of sociological mumbo-jumbo, and is now rather dated. There is still scope for someone to write the definitive book on quarks and QCD, but in the meantime, I suggest that you persuade your library to buy Watson's book — although you might want to think twice before buying it yourself. John Ellis is in the Theoretical Physics Division, CERN, Geneva 23 CH-1211, Switzerland. 